\title{
Desquamative Gingivitis: Protocols, Procedures and Critical Issues ${ }^{\dagger}$
}

\author{
Paola Carcieri \\ Department of Surgical Science. Oral Medicine Section, University of Turin, UNITO Lingotto Dental Institute, \\ Via Nizza 230, 10126 Turin, Italy; carcieri.paola@libero.it; Tel.: +39-339-578-4949 \\ + Presented at the XV National and III International Congress of the Italian Society of Oral Pathology and \\ Medicine (SIPMO), Bari, Italy, 17-19 October 2019.
}

Published: 10 December 2019

\section{Introduction}

Desquamative gingivitis (DG) isn't a specific disorder; it simply represents the gingival manifestation associated with some heterogeneous mucocutaneous disorders, such as oral lichen planus (OLP), mucous membrane pemphigoid (MMP), pemphigus vulgaris (PV), plasma cell gingivitis (PCG) and few others. Epithelial desquamation, erythema and erosive and/or vesciculobullous lesions on the gingiva usually characterise it. The DG appears more frequently in old women and menopause, although it can debut in young people and children. Clinically, it presents moderate pain, partly due to the deposit of plaque in gingival margin, being in some cases the first manifestation of the disease.

Usually of unknown aetiology, the most probable hypothesis is the autoimmune origin of these disease. For these reasons topical treatment has been indicated, as the use of corticosteroid in different forms and prescribed with different posologies, or also systemic drugs administration such as corticosteroids, other immunosuppressants and broad-spectrum antibiotics.

Even if this condition has been reported as non-plaque induced, effective dentogingival plaque control sometimes could resolve the gingival inflammation.

Some studies of periodontal status in patients with DG suggest that in patients MMP the gingivo-periodontal status is worse than health control, in the same case, patients with OLP and PV present deeper pockets and higher loss of the clinical attachment level [1].

A recent study revealed a variation of the "microbiota" in DG-patients with prevalence of some bacterial strains as high-risk pathogen Aggregatibacter Actinomycetemcomitans or moderate risk pathogens Eikenella Corrodens or Fusobacterium Nucleatum [2].

For these reasons, we postulated that efficient plaque control could have been helpful in treating DG.

We report the results of various prospective studies in which the purpose was to evaluate the clinical efficiency of an oral hygiene protocol in patients affected by DG [3-7].

\section{Results}

From January 2006 to August 2019 at the Oral Medicine Section, University of Turin, we followed 150 patients affected by DG as shown in Figure 1. The patients received a complete periodontal examination at baseline visit, during protocol and at final treatment, including: full mouth plaque scores (FMPS) Sillness and Loe (grading 0-1-2-3), full mouth bleeding upon probing scores (FMBS), probing pocket depth (PPD), mobility index sec Miller (grading 1-2-3), DGCS by Arduino et al. (score 0-4) and detection of perceived pain with VAS scale. All patients received nonsurgical periodontal therapy, including oral hygiene instructions, supra- and sub-gingival scaling if 
required. Oral hygiene instructions were given by the same experienced dental hygienist; she also provided thorough supra-gingival scaling with elimination of all deposits, over three visits and completion within 3 weeks. During each visit, subjects were instructed about proper oral hygiene maintenance at home. Moreover, the instructions were reinforced at each visit and personalised whenever necessary. A cohort of patients was also treated in combination with a solution of sodium iodide associated to salicylic acid (SISA). The solution was used at the end of each session, with an impregnated gauze (with $5 \mathrm{~mL}$ of the solution) applied for 15 minutes for both the dental arches, and for other 15 minutes with new gauzes. A second cohort received alternative approaches with ozone therapy. Its use in oral pathology is based on the principle of the liberation of $\mathrm{O}_{3}$ through the formation of an electromagnetic field, similar to what happens during the discharge of lightning in nature (Chapman effect). The ozone therapy has antimicrobial power (against aerobic and anaerobic bacteria, fungi, viruses), exerts a stimulation of the circulatory system with improvement of tissue oxygenation and modulation of immune cell and shows pain reduction and repeated insufflations stimulate neoangiogenesis with formation of epithelial tissue.

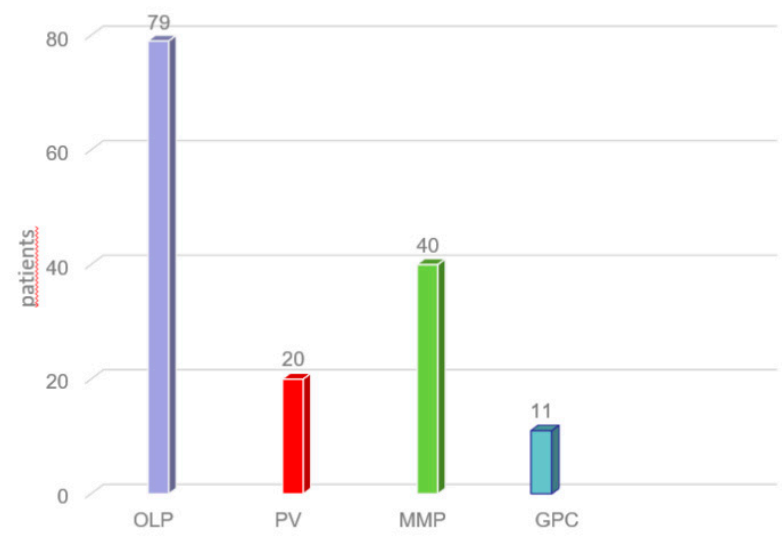

Figure 1. subdivision by type of DG of the 150 patients followed from January 2006 to August 2019 at the Oral Medicine Section, University of Turin.

In our various studies [1,3-6], we have shown that professional oral hygiene procedures are related to significant improvements of gums status, and decreases related pain, in subjects affected by autoimmune diseases as DG with specific gingival localization (Figure 2).

\begin{tabular}{l|l|l|l|l} 
& T0 & T4 & T5 & $\mathbf{p}^{\star}$ \\
\hline Full mouth bleeding score (\%) & $59.00 \pm 16.25$ & - & $23.88 \pm 11.11$ & .032 \\
\hline Full mouth plaque score (\%) & $45.02 \pm 13.63$ & - & $29.20 \pm 2.73$ & .038 \\
\hline Probing depth (mm) & $2.48 \pm 0.51$ & - & $2.29 \pm 0.67$ & .791 \\
\hline Referred symptoms (VAS score) & $5 \pm 1.32$ & $3.38 \pm 1.19$ & $2.44 \pm 1.42$ & .000 \\
\hline Activity score & $6.48 \pm 1.20$ & $3.08 \pm 1.77$ & $2.81 \pm 1.79$ & .005 \\
\hline
\end{tabular}

"Comparative statistics were performed between T0 and T5. Paired samples test was used to test the difference in

FMBS, FMPS and PPD. Wilcoxon's signed rank was used to calculate the significance of the patient related outcomes and in gingival clinical outcome (activity score).

Figure 2. results of SISA protocol reported in Carcieri P et al. [6].

\section{Conclusions}

Regular plaque and tartar removal is therefore a fundamental requirement for a favorable prognosis at a distance in DG-patients [7]. Non-surgical periodontal therapy, and oral hygiene instructions, in combination with S.I.S.A or ozone therapy, could be a possible mean in reducing 
clinical gingival inflammation and improve patient related outcomes in the different forms of DG. These protocols are conceived to recommend this as complementary line therapeutic intervention, especially in patients with only gingival involvement, during any other medical treatment, helping affected patients in maintaining a good oral hygiene.

Conflicts of Interest: The author declares no conflict of interest. The funding sponsors had no role in the design of the study; in the collection, analyses, or interpretation of data; in the writing of the manuscript, and in the decision to publish the results.

\section{References}

1. Arduino, P.G.; Farci, V.; D’Aiuto, F.; Carcieri, P.; Carbone, M.; Tanteri, C.; Gardino, N.; Gandolfo, S.; Carrozzo, M.; Broccoletti, R. Periodontal status in oral mucous membrane pemphigoid: Initial results of a case-control study. Oral Dis. 2011, 17, 90-94.

2. Arduino, P.G.; Romano, F.; Sasia, D.; Broccoletti, R.; Ricceri, F.; Barbui, A.M.; Brossa, S.; Cipriani, R.; Cricenti, L.; Cabras, M. Subgingival Microbiota in White Patients with Desquamative Gingivitis: A CrossSectional Study. J. Periodontol. 2017, 88, 643-650.

3. Arduino, P.G.; D'Aiuto, F.; Cavallito, C.; Carcieri, P.; Carbone, M.; Conrotto, D.; Defabianis, P.; Broccoletti, R. Professional Oral Hygiene as a Therapeutic Option for Pediatric Patients with Plasma Cell Gingivitis: Preliminary Results of a Prospective Case Series. J. Periodontol. 2011, 82, 1670-1675.

4. Arduino, P.G.; Lopetuso, E.; Carcieri, P.; Giacometti, S.; Carbone, M.; Tanteri, C.; Broccoletti, R. Professional oral hygiene treatment and detailed oral hygiene instruction in patients affected by mucosa membrane pemphigoid with specific gingival localization in 12 patients. Int. J. Dent. Hyg. 2012, 10, 138-141.

5. Gambino, A.; Carbone, M.; Arduino, P.G.; Carcieri, P.; Carbone, L.; Broccoletti, R. Conservative approach in patients with pemphigus gingival vulgaris: A pilot study of five cases. Int. J. Dent. 2014, 2014, 747506, doi:10.1155/2014/747506.

6. Carcieri, P.; Broccoletti, R.; Giacometti, S.; Gambino, A.; Conrotto, D.; Cabras, M.; Arduino, P.G. Favourably effective formulation of sodium iodide and salicylic acid plus professional hygiene in patients affected by desquamative gingivitis. Biol. Regul. Homeost. Agents 2016, 30, 1141-1145.

7. García-Pola Vallejo, M.J.; Rodriguez-López, S.; Fernánz-Vigil, A.; Bagán Debón, L.; García Martín, J.M. Oral hygiene instructions and professional control as part of the treatment of desquamative gingivitis. Syst. Med. Oral Patol. Oral Cir. Bucal. 2019, 24, e136-e144.

(c) 2019 by the authors. Licensee MDPI, Basel, Switzerland. This article is an open access article distributed under the terms and conditions of the Creative Commons Attribution (CC BY) license (http://creativecommons.org/licenses/by/4.0/). 